\title{
Analysis of Left Ventricular Wall Shear Stress during Diastole in Normal Subjects by Vector Flow Mapping
}

\section{Li-ping Dong}

Shanghai Sixth People's Hospital

\section{Zhen-zhen Wang}

Zhejiang Provincial People's Hospital

Hai-ru Li

Second Affiliated Hospital of Harbin Medical University

Wei-dong Yu

Second Affiliated Hospital of Harbin Medical University

jiawei tian ( $\sim$ jwtian2004@163.com )

Second Affiliated Hospital of Harbin Medical University

\section{Research Article}

Keywords: Echocardiography, Vector flow mapping, Wall shear Stress, Hemodynamics, Left ventricular

Posted Date: November 1st, 2021

DOI: https://doi.org/10.21203/rs.3.rs-969072/v1

License: (c) (i) This work is licensed under a Creative Commons Attribution 4.0 International License. Read Full License 


\section{Abstract}

Objective To observe the diastolic wall shear stress (WSS) pattern of the left ventricle (LV) by using vector flow mapping (VFM) in normal subjects.

Methods A total of 371 healthy volunteers were recruited into this study and divided into four age groups. The LV WSS was measured at each diastolic phase, and the mapping of WSS was analyzed.

Results Among groups I, II and III, The absolute value of WSS of Anterolateral \Inferoseptal and Anterospetal segments in phase D1区WSS values of inferolateral \Inferoseptal and Anterospetal segments in phase D4 all showed an increasing trend with age. In terms of gender differences, In most cases $\rrbracket$ women had greater diastolic WSS values compared to men. For each age group, the log-transformed WSS value appeared the increasing-decreasing-increasing trend from phase D1 to D4, with a peak value at the rapid filling phase.Multivariate backward stepwise linear regression analysis revealed that the certain segments log-transformed WSS was independently related to conventional parameters in evaluating diastolic function $\llbracket$ mitral lateral E/e', septal E/e', mitral lateral e', septal e' and LAVI区.

Conclusions In diastolic period, segmental LV WSS shows a regular variation phenomenon and has specific age- and gender-related patterns in different diastolic phases. The mapping of WSS may help identify the diastolic hemodynamic changes or diastolic function phase by phase.

\section{Introduction}

In the past few years, increasing attention has been dedicated to the intraventricular flow pattern. Flow patterns is the consequence of the heart's chiral geometry and the interaction of the filling jet with the walls and mitral valve of the left ventricle (LV), and they reveal the exceptional adaptability of the cardiovascular system for maintaining relatively constant blood circulation under a high workloads[1, 2]. The abnormal flow patterns within the ventricular chamber is related to many sorts of LV dysfunction, such as myocardial ischemia[3], cardiomyopathy[4], and thrombosis[5]. Therefore, flow patterns may offer a novel index of LV dysfunction[6].

Vector flow mapping (VFM), which is a combination with the color Doppler and two-dimensional speckle tracking technique, is a novel echocardiographic technology that can visualize the intraventricular flow patterns[7]. Blood flow visualization studies provide clues to reveal physiological and pathophysiological mechanisms by which abnormal flow patterns increase cardiac workload and deteriorate ventricular functions $[8,9]$. Energy loss (EL), circle, and wall shear stress (WSS), which derived from the intraventricular flow velocity vector field, are parameters that reflect the spatial dispersion of intraventricular flow patterns. Most previous studies on VFM focused on EL and circle[3, 10]. But recently, WSS has been reported to be a new quantification parameter[11], because it may indicate the underlying association between fluid mechanics and cardiovascular diseases (CVDs)risk [12]. WSS plays a key role in regulating endocardial cells and triggers a series of biological signal transduction, which in turn, regulates gene expression and function of vascular wall cells[13, 14]. For example, in the embryonic 
stage, WSS can influence the development of the original heart by adjusting the endocardial cells. High blood flow shear force is beneficial to maintain normal vascular endothelial function, and low blood flow shear force often indicates that endothelial function is damaged[15, 16].

Although WSS has been reported useful in previous studies, litter was known about its value in evaluating diastolic functions. This study aimed to explore the LV WSS variation in the diastolic period and analyze the differences stratified by age and gender. Furthermore, to provide a quantitative evaluation of the diastolic LV intracavity blood flow and function.

\section{Methods}

\section{Study population}

A total of 371 healthy volunteers (60.6\% women), mean (SD) age was $43(17)$, were recruited from the physical examination center of the Second Affiliated Hospital of Harbin Medical University from August 2018 to March 2019. Afterward, they were separated into four groups according to their age quartiles. Including criteria were normal at clinical presentation, no history of any comorbidity, and ECG of sinus rhythm.All subjects provided informed consent for study participation and anthracycline therapy administration. This study was approved by the ethics committee ofthe Second Affiliated Hospital of Harbin Medical University.Conflictsofinterest $\mathbb{X}$ The authors have no conflicts of interest to declare.

\section{Image acquisition}

The study was performed on Hitachi Aloka LISENDO 880 ultrasound system (Hitachi-Aloka Medical, Ltd, Tokyo, Japan), with the phased array single crystal probe(probe frequency $1.0-5.0 \mathrm{MHz}$, frame rate $66-78$ $\mathrm{Hz}$ ). All subjects were connected to a 12-lead ECG and examined in the left lateral decubitus position. Conventional and tissue Doppler transthoracic echocardiography (TTE) was performed by experienced sonographers and reviewed by senior physicians. In two-dimensional (2D) echocardiographic assessment, left ventricular end-diastolic diameter (LVEDd), left ventricular end-systolic diameter (LVESd), end-diastolic septal thickness (IVST), and left ventricular posterior wall thickness (LVPWT) were measured. In PW/TDI evaluation, the early diastolic peak velocity (E) and late diastolic peak velocity (A) of the mitral valve were measured in the apical four-chamber view, with the E/A ratio calculated. The early diastolic peak velocity of mitral annulus (lateral e' and septal e') was also measured for further calculation of lateral E/e' and septal E/e'. The left ventricular ejection fraction (LVEF) was generated by the biplane Simpson method. In terms of VFM mode, the 2D gain was adjusted to optimized visualization of the endocardium, mitral valve, and aortic valve. The size of sampling frame was also adjusted to completely envelop the LV, while the Nyquist limit for CDFI was set high enough so that the flow filled the left ventricle without aliasing and blood flow spilling. The 2D and CDFI images of the apical 4-chamber (Apic 4C), 3-chamber (Apic 3C), and 2-chamber (Apic 2C) views were recorded (Fig. 1). 


\section{Data analysis}

The acquired images were then imported into the DAS-RS1 workstation for offline analysis. Firstly, the endocardial border was traced to the clearest frame manually, and then the software automatically traced to the remaining frames. The user was allowed to check and edit the image frame by frame. The diastolic period was defined as the first frame after aortic valve closure to the first frame after mitral valve closure. And the diastolic period was then divided into four phases based on the ECG, time-flow curve, and twodimensional cardiac valvular opening and closing, including isovolumic diastolic period (D1), rapid filling period (D2), slow filling period (D3), and atrial contraction period (D4).

The WSS images, together with the raw data of the Apic 4C, 3C, and2C, were processed on the offline VFM workstation, and the raw data were subsequently imported into the WSS segmentation template [see Additional file 1], in which LV was divided into six walls: anterior, anterolateral, inferolateral, inferior, inferoseptal and anteroseptal (Fig. 2). Since the VFM technique is a combination of CDFI and twodimensional speckle tracking, it could acquire both the radial and axial flow velocity, as well as the boundary conditions of bilateral walls, allowing the continuity equation to calculate the intraventricular flow quantitatively (Figure 3). The Newton inner friction equation of WSS was as follows[17]:

\section{WSS『 $\mu(V \max -0) / d y \rrbracket \mu \max / d y$}

$\mu$ : blood viscosity coefficient $4.0 \times 10^{-3}\left(\mathrm{~N} \square \mathrm{s} \square \mathrm{m}^{-2}\right)$

In the equation, Vmax means the near-wall maximum flow velocity in the cardiac chamber, and the dy means the distance between the point of Vmax and the adjacent ventricular wall. As such, the (Vmax$0)$ /dy represents the gradient of near-wall flow velocity. And WSS, which is a vector with size and direction, is the product of blood viscosity $(\mu)$ and $(V \max -0) / d y[18,19]$.

\section{Statistical analysis}

The SPSS statistic software (IBM SPSS, version 22.0, Chicago, IL) was applied.Continuous data were expressed as mean \pm standard deviation (SD), and those with skewed distributions are presented as medians

(25th and 75th percentiles).Comparisons among different age groups were analyzed using the Kolmogorov-Smirnov test Comparisons between men and women were carried out by the Mann-Whitney tests. Moreover, Log-transformed WSS value in the single LV wall among different phases were compared by one-way repeated measures analysis of variance (ANOVA).The independent correlations between logtransformed WSS and conventional parameters in evaluating diastolic function were explored using stepwise multiple regressions. $\mathrm{P}<0.05$ was considered statistically significant. 
The study was approved by the Harbin Medical University and was conducted in accordance with the principles of the Declaration of Helsinki

\section{Results}

\section{Clinical and conventional echocardiographic parameters}

A total of 375 healthy subjects that satisfied the inclusion criteria were recruited originally for this study. However, four were excluded because of the poor image quality, and finally, 371 entered the analysis. The clinical data and echocardiographic measurements of all eligible subjects were listed based on different age groups in Table 1. Peak A velocity of mitral inflow was increased with age, whereas peak $E$ velocity of mitral inflow, lateral e' and septal e' velocity of mitral annulus, E/A, mitral lateral E/e' and septal E/e' decreased with age $(P<0.05)$. 
Table 1

Clinical and echocardiographic measurements of the study populations

\begin{tabular}{|c|c|c|c|c|c|}
\hline & $\begin{array}{l}\text { Group } 见 \\
(\mathrm{~N}=116)\end{array}$ &  & $\begin{array}{l}\text { Group } \\
(\mathrm{N}=86)\end{array}$ & 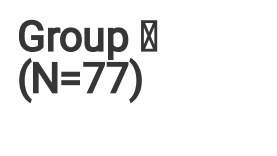 & $P$ \\
\hline Age, yrs & $22.50 \pm 3.17$ & $37.59 \pm 3.83$ & $50.31 \pm 3.88$ & $62.35 \pm 3.79$ & \\
\hline $\mathrm{BMI}, \mathrm{kg} / \mathrm{m}^{2}$ & $21.71 \pm 3.02$ & $23.11 \pm 4.17$ & $23.79 \pm 2.69$ & $23.01 \pm 2.71$ & 0.042 \\
\hline Heartrate, beats/min & $72.00 \pm 10.00$ & $76.00 \pm 10.00$ & $73.00 \pm 9.00$ & $80.00 \pm 7.00$ & $<0.001$ \\
\hline LVEDD, mm & $42.82 \pm 4.04$ & $44.70 \pm 3.90$ & $43.59 \pm 3.85$ & $44.43 \pm 4.34$ & 0.004 \\
\hline LVEDS, mm & $25.17 \pm 3.68$ & $24.78 \pm 3.54$ & $23.14 \pm 4.00$ & $23.62 \pm 3.52$ & $<0.001$ \\
\hline IVST, mm & $8.62 \pm 1.96$ & $9.32 \pm 1.25$ & $9.34 \pm 1.51$ & $8.90 \pm 1.81$ & 0.005 \\
\hline LVPWT, mm & $8.51 \pm 1.29$ & $9.16 \pm 1.24$ & $9.31 \pm 1.29$ & $8.90 \pm 1.26$ & $<0.001$ \\
\hline LAVI, $\mathrm{cm}^{3}$ & $8.63 \pm 2.69$ & $10.14 \pm 3.01$ & $9.87 \pm 3.67$ & $11.32 \pm 3.78$ & $<0.001$ \\
\hline PAD, mm & $19.29 \pm 2.73$ & $20.05 \pm 2.23$ & $20.04 \pm 2.80$ & $20.47 \pm 2.45$ & 0.013 \\
\hline LVEF,\% & $67.37 \pm 3.51$ & $67.31 \pm 3.99$ & $67.25 \pm 3.92$ & $66.98 \pm 4.14$ & 0.920 \\
\hline $\begin{array}{l}\text { Peak E-wave velocity, } \\
\mathrm{cm} / \mathrm{sec}\end{array}$ & $83.65 \pm 17.03$ & $76.37 \pm 18.08$ & $69.63 \pm 16.99$ & $68.12 \pm 15.29$ & $<0.001$ \\
\hline $\begin{array}{l}\text { Peak A-wave velocity, } \\
\mathrm{cm} / \mathrm{sec}\end{array}$ & $47.56 \pm 12.05$ & $54.43 \pm 12.84$ & $60.47 \pm 15.13$ & $72.66 \pm 16.96$ & $<0.001$ \\
\hline MV E/A ratio & $1.87 \pm 0.64$ & $1.44 \pm 0.34$ & $1.25 \pm 0.72$ & $0.97 \pm 0.28$ & $<0.001$ \\
\hline Mitral lateral e', cm/s & $18.37 \pm 3.89$ & $14.43 \pm 3.02$ & $11.68 \pm 2.77$ & $10.53 \pm 2.29$ & $<0.001$ \\
\hline Mitral lateral E/e' & $-4.73 \pm 1.24$ & $-5.45 \pm 1.40$ & $-6.07 \pm 1.64$ & $-6.64 \pm 1.55$ & $<0.001$ \\
\hline Mitral septal e', cm/s & $12.62 \pm 2.27$ & $10.95 \pm 7.80$ & $8.28 \pm 1.91$ & $7.58 \pm 1.62$ & $<0.001$ \\
\hline Mitral septal E/e' & $-6.54 \pm 1.34$ & $-7.24 \pm 3.12$ & $-8.44 \pm 2.01$ & $-9.09 \pm 2.02$ & $<0.001$ \\
\hline Tei index & $0.35 \pm 0.04$ & $0.36 \pm 0.05$ & $0.38 \pm 0.11$ & $0.35 \pm 0.05$ & 0.027 \\
\hline $\mathrm{Vp}, \mathrm{cm} / \mathrm{s}$ & $77.59 \pm 14.26$ & $76.02 \pm 16.12$ & $76.29 \pm 15.64$ & $74.03 \pm 18.59$ & 0.513 \\
\hline $\mathrm{E} / \mathrm{Vp}$ & $0.11 \pm 0.03$ & $0.10 \pm 0.03$ & $0.10 \pm 0.03$ & $0.10 \pm 0.04$ & 0.002 \\
\hline \multicolumn{6}{|c|}{$\begin{array}{l}\text { Measurements are shown as means } \pm \text { SD; BMI, Body Mass Index; BSA, body surface area; LVEDD, left } \\
\text { ventricular end-diastolic diameter; LVESD, left ventricular end-systolic diameter; IVS, interventricular } \\
\text { septum; LVPW, left posterior ventricular wall; LAVI, left atrial volume index; PAD, pulmonary artery } \\
\text { diameter; Vp, propagation velocity of early diastolic mitral inflow; and LVEF, left ventricular ejection } \\
\text { fraction; } P<0.05 \text { :The difference is statistically significant. }\end{array}$} \\
\hline
\end{tabular}




\section{WSS characteristics in different ages/Genders}

Segmental WSS values had significant phase-related differences in each age group, such as WSS of Anterior $\square$ Anterolateral Inferior and Inferoseptal segments in each diastolic phase,WSS of Anterospetal in phase D1, D3and D4.WSS of inferolateral segments in phase D2, D3and D4. Besides, WSS of Anterolateral,Inferoseptal and Anterospetal segments in phase D1;WSS of inferolateral IInferoseptal and Anterospetal segments in phase D4 all showed an increasing trend with age among groups I, II and III $(P<0.05$, Table 2). Segmental/phase gender differences of WSS could be found. One case[Women had greater diastolic WSS than men in anterior and anterolateral segments of phase D2 Linferoseptal segments of phase D3 and anterolateral segment of phase D4. and the other casea, men had greater diastolic WSS than women in inferoseptal and anterospetal segment in phase $D 4$. ( $P<0.05$, Table 3$)$. 
Table 2

The LV WSS values in the diastolic period stratified by different age groups

\begin{tabular}{|c|c|c|c|c|c|c|}
\hline \multicolumn{2}{|c|}{ WSS values } & $\begin{array}{l}\text { Group } \rrbracket \\
(\mathrm{N}=116)\end{array}$ & \multirow{2}{*}{  } & \multirow{2}{*}{ 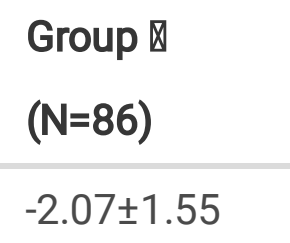 } & \multirow{2}{*}{ 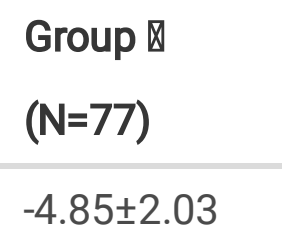 } & \multirow{2}{*}{$\begin{array}{l}P \\
<0.001\end{array}$} \\
\hline D1 & Anterior & $-2.80 \pm 1.78$ & & & & \\
\hline & Anterolateral & $-2.51 \pm 0.87$ & $-3.28 \pm 1.98$ & $-3.52 \pm 2.62$ & $-3.85 \pm 2.35$ & $<0.001$ \\
\hline & Inferolateral & $3.80 \pm 2.55$ & $4.61 \pm 3.24$ & $3.33 \pm 2.49$ & $4.17 \pm 2.29$ & 0.028 \\
\hline & Inferior & $2.59 \pm 1.48$ & $3.62 \pm 2.73$ & $4.13 \pm 2.55$ & $3.16 \pm 1.90$ & $<0.001$ \\
\hline & Inferoseptal & $1.60 \pm 1.34$ & $2.46 \pm 1.37$ & $2.80 \pm 1.89$ & $3.40 \pm 2.22$ & $<0.001$ \\
\hline & Anterospetal & $-1.89 \pm 1.42$ & $-2.19 \pm 1.45$ & $-2.59 \pm 1.90$ & $-3.79 \pm 1.98$ & $<0.001$ \\
\hline \multirow[t]{6}{*}{ D2 } & Anterior & $-33.64 \pm 19.60$ & $-30.74 \pm 22.60$ & $-46.36 \pm 31.64$ & $-53.90 \pm 35.74$ & $<0.001$ \\
\hline & Anterolateral & $-25.82 \pm 16.71$ & $-11.09 \pm 5.94$ & $-28.50 \pm 17.09$ & $-36.81 \pm 25.45$ & $<0.001$ \\
\hline & Inferolateral & $16.42 \pm 12.81$ & $21.76 \pm 15.24$ & $35.72 \pm 20.54$ & $25.15 \pm 17.40$ & $<0.001$ \\
\hline & Inferior & $25.62 \pm 14.47$ & $42.90 \pm 29.86$ & $26.48 \pm 14.64$ & $23.57 \pm 16.11$ & $<0.001$ \\
\hline & Inferoseptal & $31.44 \pm 22.39$ & $24.95 \pm 17.78$ & $22.62 \pm 10.80$ & $22.83 \pm 11.78$ & 0.006 \\
\hline & Anterospetal & $-31.35 \pm 20.69$ & $-33.06 \pm 28.17$ & $-34.47 \pm 24.80$ & $-31.80 \pm 22.62$ & 0.963 \\
\hline \multirow[t]{6}{*}{ D3 } & Anterior & $-2.25 \pm 1.13$ & $-2.08 \pm 0.88$ & $-9.44 \pm 4.99$ & $-2.09 \pm 1.12$ & $<0.001$ \\
\hline & Anterolateral & $-2.95 \pm 2.20$ & $-0.44 \pm 2.28$ & $-1.77 \pm 1.20$ & $-1.81 \pm 0.87$ & $<0.001$ \\
\hline & Inferolateral & $3.20 \pm 2.10$ & $4.22 \pm 2.59$ & $2.09 \pm 1.26$ & $2.46 \pm 1.69$ & $<0.001$ \\
\hline & Inferior & $2.39 \pm 1.54$ & $-2.51 \pm 1.82$ & $0.81 \pm 0.58$ & $2.16 \pm 1.50$ & $<0.001$ \\
\hline & Inferoseptal & $1.75 \pm 1.60$ & $2.13 \pm 1.16$ & $1.12 \pm 0.94$ & $2.01 \pm 1.49$ & $<0.001$ \\
\hline & Anterospetal & $-4.71 \pm 3.31$ & $-1.39 \pm 0.99$ & $-1.39 \pm 0.91$ & $-1.96 \pm 1.25$ & $<0.001$ \\
\hline \multirow[t]{6}{*}{ D4 } & Anterior & $-4.49 \pm 2.87$ & $-10.77 \pm 5.52$ & $-25.81 \pm 16.81$ & $-25.42 \pm 14.99$ & $<0.001$ \\
\hline & Anterolateral & $-9.44 \pm 5.41$ & $-10.53 \pm 6.93$ & $-24.63 \pm 18.25$ & $-19.80 \pm 10.75$ & $<0.001$ \\
\hline & Inferolateral & $9.48 \pm 4.95$ & $15.80 \pm 8.60$ & $16.54 \pm 8.59$ & $17.10 \pm 9.80$ & $<0.001$ \\
\hline & Inferior & $8.98 \pm 3.56$ & $14.94 \pm 6.30$ & $22.83 \pm 12.47$ & $21.89 \pm 19.27$ & $<0.001$ \\
\hline & Inferoseptal & $9.39 \pm 4.54$ & $11.17 \pm 5.46$ & $14.53 \pm 4.90$ & $14.66 \pm 5.61$ & $<0.001$ \\
\hline & Anterospetal & $-7.73 \pm 4.45$ & $-9.14 \pm 3.52$ & $-11.85 \pm 5.58$ & $-12.48 \pm 8.26$ & $<0.001$ \\
\hline
\end{tabular}


Table 3

Differences in LV WSS values between men and women at diastolic period

\begin{tabular}{|c|c|c|c|c|}
\hline \multicolumn{2}{|c|}{ WSS values } & \multirow{2}{*}{$\begin{array}{l}\text { Men } \\
(\mathrm{N}=146) \\
-3.56(3.93)\end{array}$} & \multirow{2}{*}{$\begin{array}{l}\text { Women } \\
(\mathrm{N}=225) \\
-3.09(3.46)\end{array}$} & \multirow{2}{*}{$\begin{array}{l}P \\
0.257\end{array}$} \\
\hline D1 & Anterior & & & \\
\hline & Anterolateral & $-2.95(2.62)$ & $-2.85(2.54)$ & 0.671 \\
\hline & Inferolateral & $4.21(4.20)$ & $3.56(3.44)$ & 0.128 \\
\hline & Inferior & $3.32(3.64)$ & $3.15(2.96)$ & 0.838 \\
\hline & Inferoseptal & $2.11(2.59)$ & $2.32(2.33)$ & 0.839 \\
\hline & Anterospetal & $-2.32(2.56)$ & $-2.38(2.65)$ & 0.686 \\
\hline \multirow[t]{6}{*}{ D2 } & Anterior & $-30.21(33.86)$ & $38.93(41.25)$ & 0.021 \\
\hline & Anterolateral & $-17.03(19.86)$ & $-23.08(28.27)$ & $<0.001$ \\
\hline & Inferolateral & $20.48(20.82)$ & $21.79(22.47)$ & 0.819 \\
\hline & Inferior & $23.28(25.13)$ & $26.73(21.84)$ & 0.820 \\
\hline & Inferoseptal & 22.07(18.12) & $24.75(19.43)$ & 0.325 \\
\hline & Anterospetal & $-25.77(32.48)$ & $-31.58(30.87)$ & 0.142 \\
\hline \multirow[t]{6}{*}{ D3 } & Anterior & $-2.33(1.96)$ & $-2.44(2.38)$ & 0.679 \\
\hline & Anterolateral & $-1.68(2.09)$ & $-1.92(2.13)$ & 0.059 \\
\hline & Inferolateral & $3.11(3.62)$ & $2.77(2.31)$ & 0.114 \\
\hline & Inferior & $0.70(2.86)$ & $1.09(2.54)$ & 0.06 \\
\hline & Inferoseptal & $1.34(1.67)$ & $1.75(1.94)$ & 0.025 \\
\hline & Anterospetal & $-1.95(2.39)$ & $-1.82(2.44)$ & 0.657 \\
\hline \multirow[t]{6}{*}{ D4 } & Anterior & $-9.07(13.68)$ & $-11.58(21.78)$ & 0.199 \\
\hline & Anterolateral & $-11.17(11.83)$ & $-13.76(15.40)$ & 0.019 \\
\hline & Inferolateral & 13.18(10.33) & $12.08(12.07)$ & 0.116 \\
\hline & Inferior & $13.79(11.43)$ & $12.93(16.61)$ & 0.281 \\
\hline & Inferoseptal & $12.98(7.06)$ & 11.39(7.54) & 0.011 \\
\hline & Anterospetal & $-10.38(5.81)$ & $-8.58(7.49)$ & 0.008 \\
\hline
\end{tabular}




\section{WSS characteristics in different diastolic phases}

For each age group, there were significant differences in the log-transformed LV WSS value among different diastolic phases.And the absolute value of the log-transformed WSS appeared the increasingdecreasing--increasing trend from phase D1 to D4, with a peak value at the rapid filling phase (Fig. 4).

\section{Association between WSS and conventional parameters in evaluating diastolic function}

Multiple regression analysis was conducted to evaluate the association between diastolic logtransformed LV WSS and the 2D echocardiographic determiners of diastolic function (Tables 4 and 5). After adjusting for age and gender, Inferior WSS in phase D2,Anterospetal WSS in phase D3 and Anterior and Anterolateral WSS in phase D4 are independently associated with lateral E/e'. Anterolateral WSS in phase D2 $\square$ Anterospetal WSS in phase D3 and Anterior $\square$ Anterolateral and Anterospetal WSS in phase D4 are independently associated with septal E/e'.Anterolateral,Inferoseptal and Anterospetal WSS in phase D1 ;Inferolateral and Inferior WSS in phase D2־Anterior ,Anterolateral ,Inferolateral and Anterospetal WSS in phase D3ロall segments WSS in phase D4 are all independently associated with lateral e'. Anterolateral WSS in phase D3 had an independent association with septal e'. Anterior WSS in phase D1 had an independent correlation with LAVI $(P<0.05)$. 
Table 4

Multiple regression analysis of the LV WSS with $\mathrm{E} / \mathrm{e}^{\prime}$

\begin{tabular}{|c|c|c|c|c|}
\hline & Mitral Lateral E/e' & & Mitral Septal E/e' & \\
\hline & Standardized $\beta$ Coefficient & $P$ & Standardized $\beta$ Coefficient & $P$ \\
\hline Age & -0.444 & $<0.001$ & -0.314 & $<0.001$ \\
\hline Gender & -0.074 & 0.134 & -0.108 & 0.034 \\
\hline $\mathrm{BMI}$ & 0.037 & 0.431 & 0.049 & 0.305 \\
\hline \multicolumn{5}{|l|}{ WSS } \\
\hline D1 Anterior & -0.015 & 0.865 & -0.019 & 0.844 \\
\hline Anterolateral & -0.120 & 0.182 & -0.089 & 0.341 \\
\hline Inferolateral & 0.152 & 0.093 & 0.106 & 0.261 \\
\hline Inferior & -0.025 & 0.782 & -0.022 & 0.818 \\
\hline Inferoseptal & -0.050 & 0.568 & 0.127 & 0.169 \\
\hline Anterospetal & 0.075 & 0.395 & -0.036 & 0.699 \\
\hline D2 Anterior & 0.067 & 0.456 & 0.184 & 0.050 \\
\hline Anterolateral & 0.037 & 0.680 & 0.206 & 0.029 \\
\hline Inferolateral & 0.067 & 0.456 & -0.159 & 0.090 \\
\hline Inferior & 0.185 & 0.043 & -0.058 & 0.546 \\
\hline Inferoseptal & 0.060 & 0.512 & -0.050 & 0.604 \\
\hline Anterospetal & -0.028 & 0.764 & 0.017 & 0.863 \\
\hline D3 Anterior & -0.032 & 0.716 & 0.057 & 0.535 \\
\hline Anterolateral & 0.153 & 0.086 & -0.101 & 0.276 \\
\hline Inferolateral & 0.001 & 0.993 & -0.048 & 0.605 \\
\hline Inferior & -0.073 & 0.427 & 0.047 & 0.623 \\
\hline Inferoseptal & -0.165 & 0.070 & 0.030 & 0.751 \\
\hline Anterospetal & 0.196 & 0.025 & -0.222 & 0.016 \\
\hline D4 Anterior & -0.198 & 0.012 & 0.303 & $<0.001$ \\
\hline Anterolateral & -0.293 & 0.001 & 0.343 & $<0.001$ \\
\hline Inferolateral & 0.030 & 0.731 & -0.089 & 0.336 \\
\hline
\end{tabular}




\begin{tabular}{|lllll|}
\hline & Mitral Lateral E/e' & & Mitral Septal E/e' & \\
\hline Inferior & 0.155 & 0.069 & -0.063 & 0.476 \\
\hline Inferoseptal & 0.162 & 0.062 & -0.044 & 0.624 \\
\hline Anterospetal & -0.046 & 0.598 & 0.205 & 0.025 \\
\hline
\end{tabular}


Table 5

Multiple regression analysis of the LV WSS with other evaluation indexs of diastolic function

Mitral Lateral e'

Standardized $\beta \quad P$

Coefficient
Mitral Septal e'

Standardized $\beta \quad P$

Coefficient
LAVI

Standardized $P$ $\beta$ Coefficient

$\begin{array}{lllllll}\text { Age } & -0.678 & <0.001 & -0.355 & <0.001 & 0.275 & <0.001 \\ \text { Gender } & 0.063 & 0.091 & 0.019 & 0.707 & -0.227 & <0.001 \\ \text { BMI } & 0.050 & 0.155 & 0.013 & 0.784 & -0.004 & 0.945\end{array}$

WSS

\begin{tabular}{lllllll|} 
D1 Anterior & 0.002 & 0.978 & 0.067 & 0.436 & -0.146 & 0.006 \\
\hline Anterolateral & 0.243 & 0.002 & 0.106 & 0.214 & -0.001 & 0.981 \\
\hline Inferolateral & -0.024 & 0.759 & -0.071 & 0.407 & -0.025 & 0.629 \\
\hline Inferior & -0.014 & 0.860 & -0.006 & 0.941 & 0.068 & 0.203 \\
\hline Inferoseptal & -0.258 & 0.001 & -0.102 & 0.220 & 0.008 & 0.873 \\
\hline Anterospetal & 0.157 & 0.044 & 0.116 & 0.170 & -0.049 & 0.341 \\
\hline D2 Anterior & 0.108 & 0.167 & -0.091 & 0.283 & 0.005 & 0.925 \\
\hline Anterolateral & 0.027 & 0.730 & -0.052 & 0.544 & 0.100 & 0.056 \\
\hline Inferolateral & -0.279 & $<0.001$ & 0.160 & 0.060 & 0.011 & 0.833 \\
\hline Inferior & -0.187 & 0.019 & 0.052 & 0.546 & 0.054 & 0.311 \\
\hline Inferoseptal & 0.031 & 0.702 & 0.084 & 0.332 & -0.006 & 0.908 \\
\hline Anterospetal & 0.075 & 0.353 & -0.020 & 0.818 & 0.018 & 0.731 \\
\hline D3 Anterior & 0.282 & $<0.001$ & 0.015 & 0.862 & 0.010 & 0.849 \\
\hline Anterolateral & -0.36 & $<0.001$ & 0.254 & 0.003 & 0.055 & 0.283 \\
\hline Inferolateral & 0.208 & 0.008 & 0.149 & 0.078 & 0.051 & 0.327 \\
\hline Inferior & 0.113 & 0.159 & -0.056 & 0.519 & -0.069 & 0.197 \\
\hline Inferoseptal & 0.058 & 0.469 & -0.046 & 0.595 & -0.095 & 0.073 \\
\hline Anterospetal & -0.400 & $<0.001$ & 0.159 & 0.056 & 0.034 & 0.504 \\
\hline D4 Anterior & 0.502 & $<0.001$ & -0.057 & 0.450 & -0.019 & 0.676 \\
\hline Anterolateral & 0.387 & $<0.001$ & -0.121 & 0.141 & 0.045 & 0.372 \\
\hline Inferolateral & -0.237 & 0.002 & 0.030 & 0.721 & 0.096 & 0.062
\end{tabular}




\begin{tabular}{|lllllll|}
\hline & Mitral Lateral e' & & Mitral Septal e' & \multicolumn{3}{c|}{ LAVI } \\
\hline Inferior & -0.390 & $<0.001$ & -0.051 & 0.531 & 0.072 & 0.144 \\
\hline Inferoseptal & -0.381 & $<0.001$ & -0.014 & 0.861 & 0.076 & 0.133 \\
\hline Anterospetal & 0.240 & 0.002 & -0.046 & 0.575 & -0.084 & 0.096 \\
\hline
\end{tabular}

\section{Discussion}

The temporal and spatial distribution of blood flow velocity contributes to providing the diagnostic and prognostic information of CVDs[20]. The blood flow interferes since the cardiac wall is not smooth and rough. A kind of viscous friction is produced when the near-wall blood flows through the wall due to the viscosity of blood. Thus it is essential to characterize and quantify the flow-wall interaction for evaluating the LV structure and function. WSS is the tangential component of wall friction, which also presents a vector field that is tangent to the surface anywhere. WSS can quantify the interaction of the intraventricular flow vector and the wall [12], thus reflecting the changes in cardiac structure and function. Despite the WSS can be measured by MRI[21,22], the clinical application of MRI is limited for its long examination duration, high costs, and low temporal resolution. Echocardiographic VFM technology is a novel visualization approach modified based on the method proposed by Garcia et al[18]. It could serve as an ideal tool to visualize the intraventricular flow vector, which may accurately evaluate the local and global hemodynamics during the left ventricular diastolic period, and reflect the corresponding left ventricular diastolic function[23]. In the VFM technology, the Gaussian filtering is employed to remove noise and the non-smooth factors, to smooth the blood flow, without producing lots of errors[18].

The absolute value of WSS in certain segments increased gradually with age, which may be due to the aging-related changes in blood viscosity and flow velocity. Due to the decrease of $\mathrm{HR}[24]$ and metabolic requirements[25], the intracardiac flow velocity decreases with the increases of age. However, the prevalence of triglycerides and LDL cholesterol was significantly increased with aging[26]. Elevated levels of plasma triglycerides and LDL cholesterol have been found associated with increase in blood viscosity[27]. Our study showed the absolute value change of WSS may be more affected by blood viscosity.There was also a gender discrepancy in WSS values. One plausible reason may be the higher level of testosterone in men, which may shorten the QT interval, and result in the shorter action potential duration[28]. Besides,compared with men, women may have a stronger cardiac response to demand. Thus, gender could present as a factor when evaluating WSS value [29]. All of the above may be the reason why women had greater diastolic WSS than men in most of all related segments in general.The structure and function of LV may change with age and may be affected by different diet, lifestyle, heredity[30]. In our study, WSS is proportional to blood viscosity and intracardiac flow velocity.

The velocity in the LV cavity was lowest compared with other diastolic phases in the period before the mitral valve opening after aortic valve closure. It then increased dramatically with the flow passed from the left atrium into the left ventricle rapidly[3]. The velocity gradually decreased when it turned to the 
outflow tract after reaching the apex. However, with the left atrium contracting, the flow entered the left ventricle again, resulting in increased flow velocity $[3,20,31]$. In the present study, the absolute value of LV WSS coincided with the increasing-decreasing-increasing trend during the D1 through D4 phases. This is because the change in WSS was related to blood flow velocity during the diastolic period. According to the WSS calculation formula, WSS also increased with the increase in the velocity changes[19].

According to the 2019 guidelines of the American Society of Echocardiography, E/e',lateral e', septal e' and LAVI were indexes for the assessment of left ventricular diastolic function in normal LVEF subjects[32]. In these four age groups of this study, WSS of some certain LV segments is independently associated with lateral E/e' and septal E/e', lateral e', septal e' and LAVI which indicated that WSS could be a new and helpful parameter to evaluate the left ventricular filling pressure and diastolic function.

It has been showed that the changes in WSS could reflect the changes in vascular and cardiac functions in some diseases[33]. Ji et al. reported the LV vortex and WSS were evaluated in patients with HCM. Their results showed that, compared with the control group, peak values of LV global WSS at rapid ejection phase, atrial contraction phase, and rapid filling phase increased in HCM patients, whereas that value of early diastolic phase decreased in HCM patients[11]. WSS is directly related to the vascular function, and the high WSS could regulate the inner diameter of a blood vessel, and inhibit the increase in blood vascular wall, thrombosis and inflammation. However, a lower WSS is known to express an atherogenic endothelial gene profile, as observed in the carotid arteries in subjects with risk factors for atherosclerosis[33]. Additionally, WSS also exerts a vital role in the cardiovascular system in hypertension patients, which is achieved by releasing bioactive molecules directly or indirectly [34,35]. Therefore, it is essential to explore the spatial and temporal distribution patterns of WSS during different phases in normal subjects.

Certain limitations should also be noted in this study. Firstly, the VFM technology can only examine the flow field vector change in the two-dimensional plane, and there is still a certain gap between the accurate measurements of the LV complex three-dimensional flow field vector change. Secondly, the current study was a preliminary study based on a small study population, while the changes in WSS must be verified in a larger and more diverse range. Thirdly, the blood cell contents are different among various individuals, which also results in the different blood viscosity coefficients [7].

To sum up, the LV WSS in normal subjects showed certain changes during the diastolic period in our current cohort, which coincided with the flow changes in LV, and might provide valuable information for the assessment of LV diastolic function. Further study needs to be performed to verify its clinical value in evaluating the diastolic function for both healthy individuals and patients with certain kinds of disease.

\section{Abbreviations}

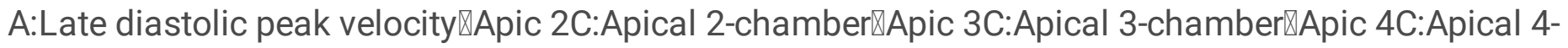

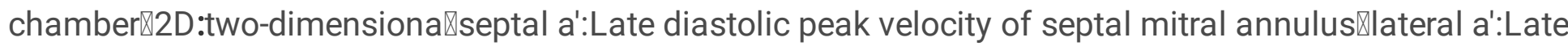

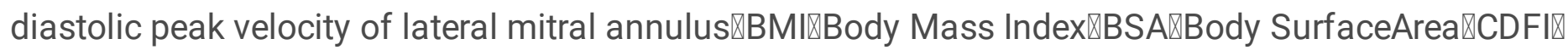




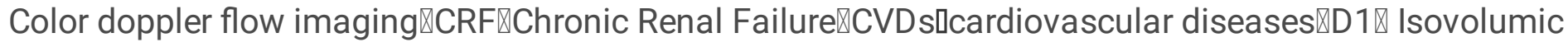
diastolic period $\triangle \mathrm{D} 2 \varangle$ Rapid filling period $\triangle \mathrm{D} 3 \triangle$ Slow filling period $\triangle \mathrm{D} 4 \varangle$ Atrial contraction period $\triangle \mathrm{E} \triangle \mathrm{E}$ Erly

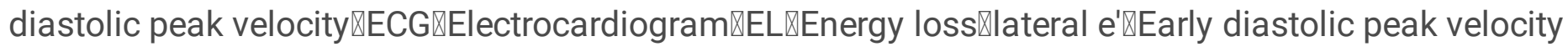

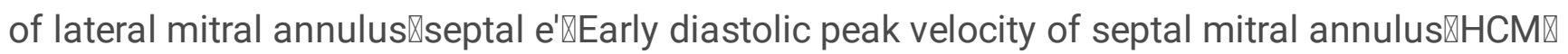

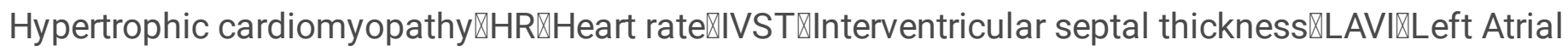

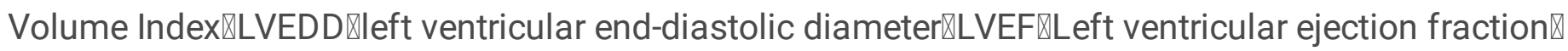

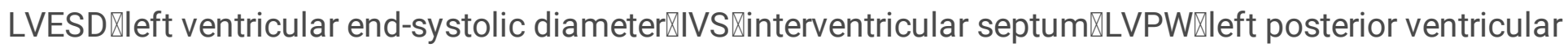

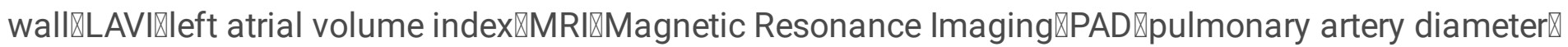
$P W \triangle P$ Pulsed wave doppler $\triangle S T I \triangle S p e c k l e$ tracking imaging $\triangle T D I \otimes T$ issue doppler imaging $\triangle T T E D$ transthoracic

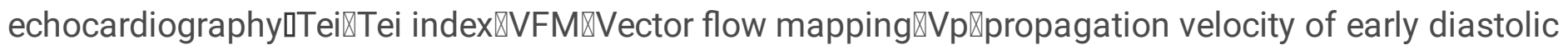
mitral inflow $₫$ WSS $₫$ Wall shear stress.

\section{Declarations}

\section{Acknowledgments}

We would like to thank all the participants for volunteering their time to be involved in this study $₫$ thank the participants from the Second Affiliated Hospital of Harbin Medical University for their help.

\section{Authors' contributions}

JWT conceived and supervised the study and performed statistical analysis of the data. LPD was involved in the procedure and wrote the main body of the manuscript. ZZW $\square$ HRL and WDY participated in the discussion on the interpretation of the research content and took part in the procedure. All authors critically revised and approved the final version of the manuscript. All authors read and approved the final manuscript.

\section{Funding}

Not applicable.

\section{Availability of data and materials}

The datasets used during the current study are available from the corresponding author on reasonable request.

\section{Ethics approval and consent to participate}


All participants provided written consent before entering the study. The study was approved by the Ethics Committee of Harbin Medical University .

\section{Consent for publication}

Not applicable.

\section{Competing interests}

All authors declare that they have no competing interests.

\section{Author details}

Li-ping Dong: Sixth people's hospital affiliated to Shanghai Jiao Tong University; Zhen-zhen Wang: Zhejiang Province People's Hospistal; Hai-ru Li: Second Affiliated Hospital of Harbin Medical University;

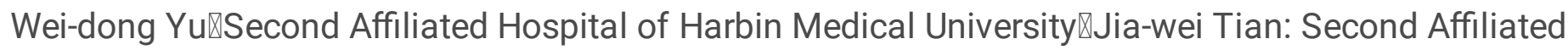
Hospital of Harbin Medical University.

\section{References}

1. Martinez-Legazpi P, Bermejo J, Benito Y, Yotti R, Perez Del Villar C, Gonzalez-Mansilla A, Barrio A, Villacorta E, Sanchez PL, Fernandez-Aviles F et al: Contribution of the diastolic vortex ring to left ventricular filling. J Am Coll Cardio/ 2014, 64(16):1711-1721.

2. Sengupta PP, Pedrizzetti G, Kilner PJ, Kheradvar A, Ebbers T, Tonti G, Fraser AG, Narula J: Emerging trends in CV flow visualization. JACC Cardiovasc Imaging 2012, 5(3):305-316.

3. Lu J, Li W, Zhong Y, Luo A, Xie S, Yin L: Intuitive visualization and quantification of intraventricular convection in acute ischemic left ventricular failure during early diastole using color Doppler-based echocardiographic vector flow mapping. Int J Cardiovasc Imaging 2012, 28(5):1035-1047.

4. Baccani B, Domenichini F, Pedrizzetti G, Tonti G: Fluid dynamics of the left ventricular filling in dilated cardiomyopathy. J Biomech 2002, 35(5):665-671.

5. Van Dantzig JM, Delemarre BJ, Bot H, Koster RW, Visser CA: Doppler left ventricular flow pattern versus conventional predictors of left ventricular thrombus after acute myocardial infarction. $J \mathrm{Am}$ Coll Cardiol 1995, 25(6):1341-1346.

6. Mohiaddin RH, Yang GZ, Kilner PJ: Visualization of flow by vector analysis of multidirectional cine MR velocity mapping. J Comput Assist Tomogr 1994, 18(3):383-392.

7. Tanaka M, Sakamoto T, Sugawara S, Nakajima H, Katahira Y, Ohtsuki S, Kanai H: Blood flow structure and dynamics, and ejection mechanism in the left ventricle: analysis using echodynamography. J Cardiol 2008, 52(2):86-101. 
8. Hong GR, Kim M, Pedrizzetti G, Vannan MA: Current clinical application of intracardiac flow analysis using echocardiography. J Cardiovasc Ultrasound 2013, 21(4):155-162.

9. Nakashima K, Itatani K, Kitamura T, Oka N, Horai T, Miyazaki S, Nie M, Miyaji K: Energy dynamics of the intraventricular vortex after mitral valve surgery. Heart Vessels 2017, 32(9):1123-1129.

10. Li CM, Bai WJ, Liu YT, Tang H, Rao L: Dissipative energy loss within the left ventricle detected by vector flow mapping in diabetic patients with controlled and uncontrolled blood glucose levels. Int $J$ Cardiovasc Imaging 2017, 33(8):1151-1158.

11. Ji L, Hu W, Yong $Y, W u ~ H, Z$, hou L, Xu D: Left ventricular energy loss and wall shear stress assessed by vector flow mapping in patients with hypertrophic cardiomyopathy. Int J Cardiovasc Imaging 2018, 34(9):1383-1391.

12. Arzani A, Shadden SC: Wall shear stress fixed points in cardiovascular fluid mechanics. $J$ Biomech 2018, 73:145-152.

13. Baeyens N, Bandyopadhyay C, Coon BG, Yun S, Schwartz MA: Endothelial fluid shear stress sensing in vascular health and disease. $J$ Clin Invest 2016, 126(3):821-828.

14. Davies PF: Hemodynamic shear stress and the endothelium in cardiovascular pathophysiology. Nat Clin Pract Cardiovasc Med 2009, 6(1):16-26.

15. Liu A, Nickerson A, Troyer A, Yin X, Cary R, Thornburg K, Wang R, Rugonyi S: Quantifying blood flow and wall shear stresses in the outflow tract of chick embryonic hearts. Comput Struct 2011, 89(1112):855-867.

16. Poelma C, Van der Heiden K, Hierck BP, Poelmann RE, Westerweel J: Measurements of the wall shear stress distribution in the outflow tract of an embryonic chicken heart. $J R$ Soc Interface 2010, 7(42):91-103.

17. Tang XS, Loke YC, Lu P, Sinha SK, O'Shea SJ: Friction measurement on free standing plates using atomic force microscopy. The Review of scientific instruments 2013, 84(1):013702.

18. Garcia D, Del Alamo JC, Tanne D, Yotti R, Cortina C, Bertrand E, Antoranz JC, Perez-David E, Rieu R, Fernandez-Aviles $\mathrm{F}$ et al: Two-dimensional intraventricular flow mapping by digital processing conventional color-Doppler echocardiography images. IEEE Trans Med Imaging 2010, 29(10):17011713.

19. Paszkowiak JJ, Dardik A: Arterial wall shear stress: observations from the bench to the bedside. Vasc Endovascular Surg 2003, 37(1):47-57.

20. Hong GR, Pedrizzetti G, Tonti G, Li P, Wei Z, Kim JK, Baweja A, Liu S, Chung N, Houle H et al: Characterization and quantification of vortex flow in the human left ventricle by contrast echocardiography using vector particle image velocimetry. JACC Cardiovasc Imaging 2008, 1(6):705-717.

21. Shojima M, Oshima M, Takagi K, Torii R, Hayakawa M, Katada K, Morita A, Kirino T: Magnitude and role of wall shear stress on cerebral aneurysm: computational fluid dynamic study of $\mathbf{2 0}$ middle cerebral artery aneurysms. Stroke 2004, 35(11):2500-2505. 
22. Xiao W, Qi T, He S, Li Z, Ou S, Zhang G, Liu X, Huang Z, Liang F: Low Wall Shear Stress Is Associated with Local Aneurysm Wall Enhancement on High-Resolution MR Vessel Wall Imaging. AJNR Am J Neuroradiol 2018, 39(11):2082-2087.

23. Uejima T, Koike A, Sawada H, Aizawa T, Ohtsuki S, Tanaka M, Furukawa T, Fraser AG: A new echocardiographic method for identifying vortex flow in the left ventricle: numerical validation. Ultrasound Med Biol 2010, 36(5):772-788.

24. Hayashi T, Itatani K, Inuzuka R, Shimizu N, Shindo T, Hirata Y, Miyaji K: Dissipative energy loss within the left ventricle detected by vector flow mapping in children: Normal values and effects of age and heart rate. J Cardiol 2015, 66(5):403-410.

25. Wu C, Honarmand AR, Schnell S, Kuhn R, Schoeneman SE, Ansari SA, Carr J, Markl M, Shaibani A: Age-Related Changes of Normal Cerebral and Cardiac Blood Flow in Children and Adults Aged 7 Months to 61 Years. J Am Heart Assoc 2016, 5(1).

26. Gu T, Zhou W, Sun J, Wang J, Zhu D, Bi Y: Gender and Age Differences in Lipid Profile Among Chinese Adults in Nanjing: a Retrospective Study of Over 230,000 Individuals from 2009 to 2015. Exp Clin Endocrinol Diabetes 2018, 126(7):429-436.

27. Irace C, Carallo C, Scavelli F, Esposito T, De Franceschi MS, Tripolino C, Gnasso A: Influence of blood lipids on plasma and blood viscosity. Clin Hemorheol Microcirc 2014, 57(3):267-274.

28. Vicente J, Johannesen L, Galeotti L, Strauss DG: Mechanisms of sex and age differences in ventricular repolarization in humans. Am Heart J 2014, 168(5):749-756.

29. Yeon SB, Salton CJ, Gona P, Chuang ML, Blease SJ, Han Y, Tsao CW, Danias PG, Levy D, O'Donnell CJ et al: Impact of age, sex, and indexation method on MR left ventricular reference values in the Framingham Heart Study offspring cohort. J Magn Reson Imaging 2015, 41(4):1038-1045.

30. Li CY, Gao BL, Guo FQ, Zhang XJ, Fan QY, Wu BL, Xiang C, Liu XW, Pan T: Quantitative evaluation of left ventricular volume and function in middle-aged healthy chinese people with 3 Tesla MRI. J Magn Reson Imaging 2016, 44(5):1143-1150.

31. Akiyama K, Maeda S, Matsuyama T, Kainuma A, Ishii M, Naito Y, Kinoshita M, Hamaoka S, Kato H, Nakajima $Y$ et al: Vector flow mapping analysis of left ventricular energetic performance in healthy adult volunteers. BMC Cardiovasc Disord 2017, 17(1):21.

32. Mitchell C, Rahko PS, Blauwet LA, Canaday B, Finstuen JA, Foster MC, Horton K, Ogunyankin KO, Palma RA, Velazquez EJ: Guidelines for Performing a Comprehensive Transthoracic Echocardiographic Examination in Adults: Recommendations from the American Society of Echocardiography. J Am Soc Echocardiogr 2019, 32(1):1-64.

33. Nigro P, Abe J, Berk BC: Flow shear stress and atherosclerosis: a matter of site specificity. Antioxid Redox Signal 2011, 15(5):1405-1414.

34. Chatzizisis YS, Coskun AU, Jonas M, Edelman ER, Feldman CL, Stone PH: Role of endothelial shear stress in the natural history of coronary atherosclerosis and vascular remodeling: molecular, cellular, and vascular behavior. J Am Coll Cardiol 2007, 49(25):2379-2393. 
35. Yang JW, Cho KI, Kim JH, Kim SY, Kim CS, You GI, Lee JY, Choi SY, Lee SW, Kim HS et al: Wall shear stress in hypertensive patients is associated with carotid vascular deformation assessed by speckle tracking strain imaging. Clin Hypertens 2014, 20:10.

\section{Figures}

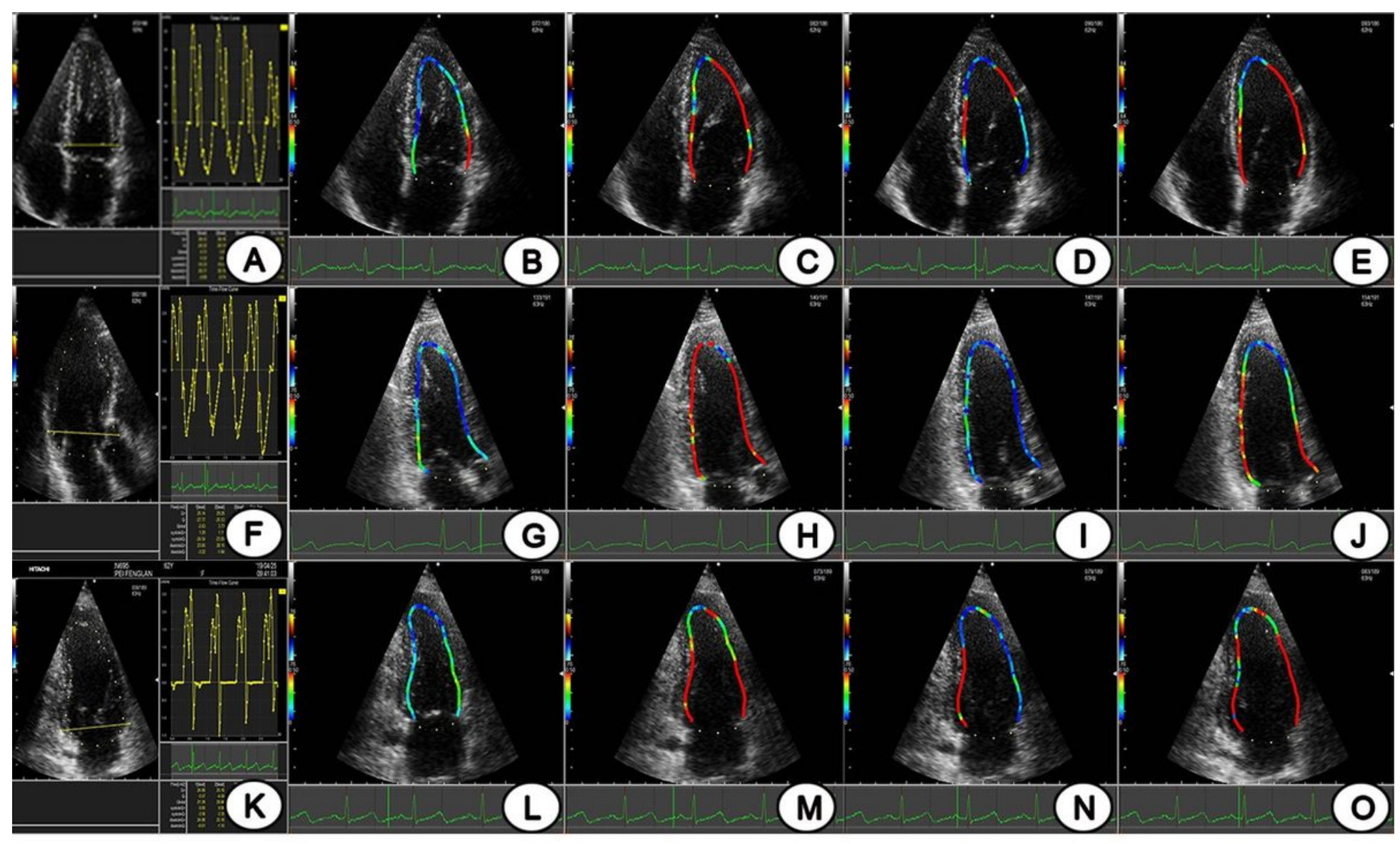

Figure 1

The LV WSS of Apic 4C,3C, and 2C; A, F and $\mathrm{K}$ are the time-flow curves of Apic 4C, 3C, and 2C; B-E:WSS; LV WSS of Apic 4C at the D1-D4 phases; G-J: LV WSS of Apic 3C at the D1-D4 phases; and L-O: LV WSS of Apic 2 C at the D1-D4 phases. 

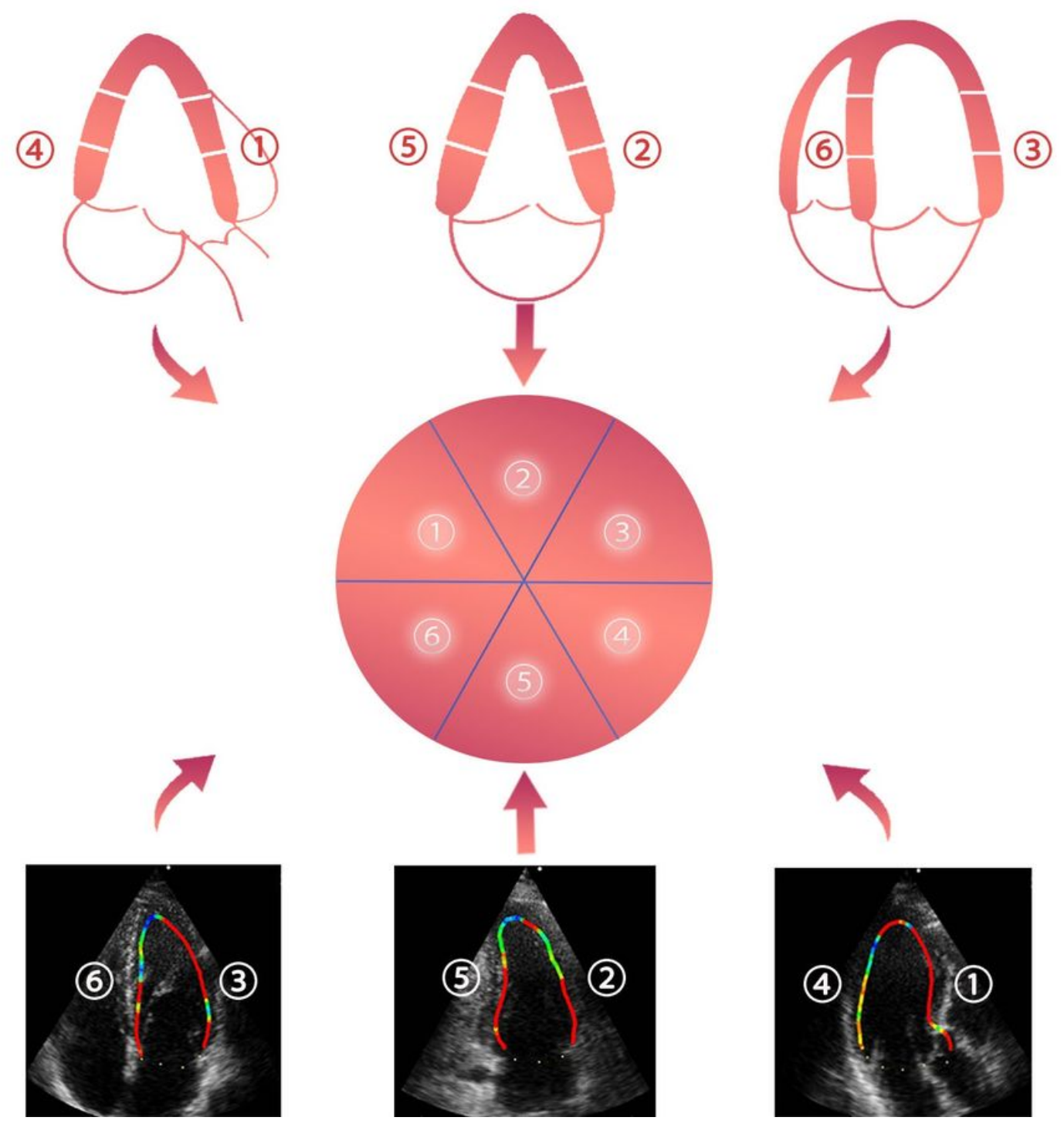

Figure 2

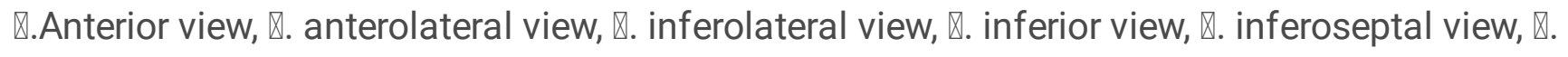
anterospetal view. 




\section{Figure 3}

The blood flow vector of the left ventricle in the apical view (3a). Vr: the blood flow vector parallel to the sound beam; $\mathrm{V} \theta$ : the blood flow vector perpendicular to the sound beam. Correlation between Vr and WSS (3b). The direction of WSS is perpendicular to the wall, from low speed to high speed.
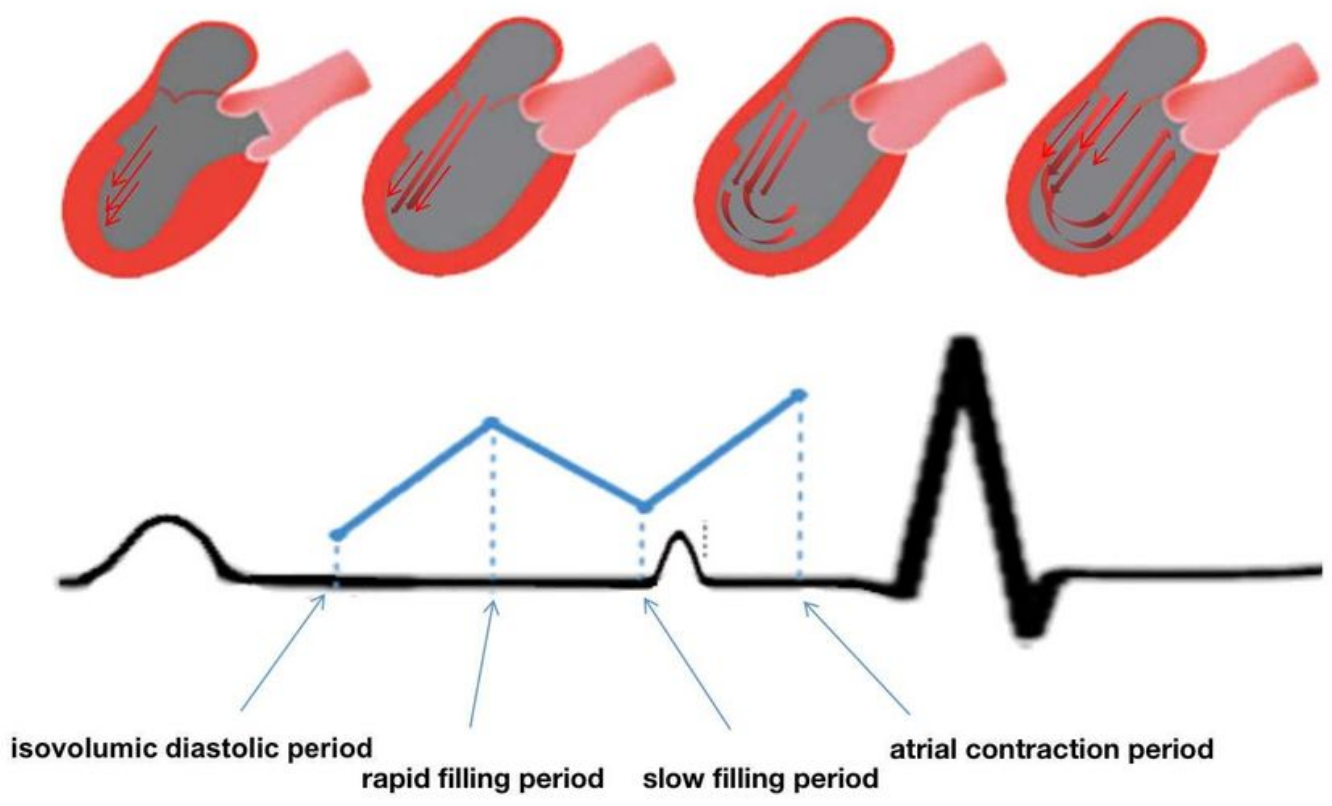

\section{Figure 4}

The variation trend of LV WSS at the diastolic period 